$$
\begin{gathered}
\text { for } \\
\text { the } \\
\text { communication } \\
\text { entitled }
\end{gathered}
$$

\title{
Sonogashira Cross-Couplings of Parent Ynamides. Syntheses of Urethane and Sulfonamide-Terminated Conjugated Phenylacetylenic Systems.
}

\author{
authored by
}

\begin{abstract}
Michael R. Tracey, Yanshi Zhang, Michael O. Frederick, Jason A. Mulder,
\end{abstract} and Richard P. Hsung*

Department of Chemistry, University of Minnesota, Minneapolis, MN 55455

\section{X-Ray Structure of}

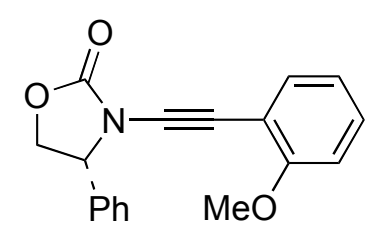


REFERENCE NUMBER: 03254a

CRYSTAL STRUCTURE REPORT

$\mathrm{C}_{18} \mathrm{H}_{15} \mathrm{~N} \mathrm{O}_{3}$

Report prepared for:

M. Tracey / Prof. R. Hsung

October 31, 2003

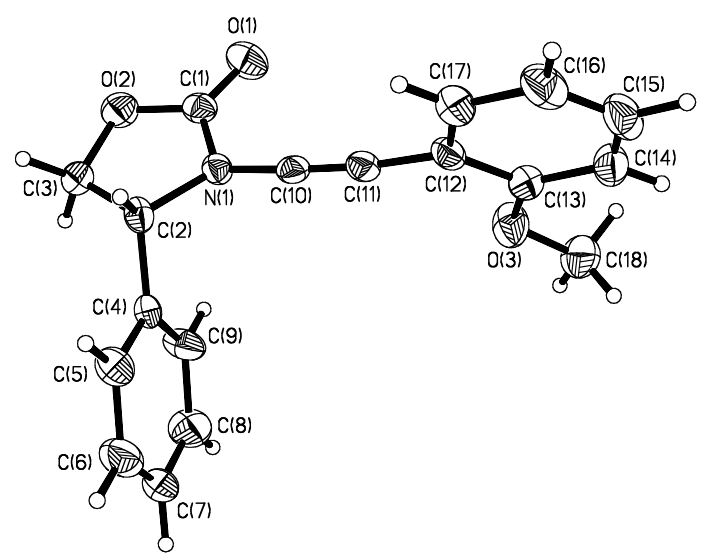

Victor G. Young, Jr

X-Ray Crystallographic Laboratory

Department of Chemistry

University of Minnesota

207 Pleasant St. S.E.

Minneapolis, MN 55455 


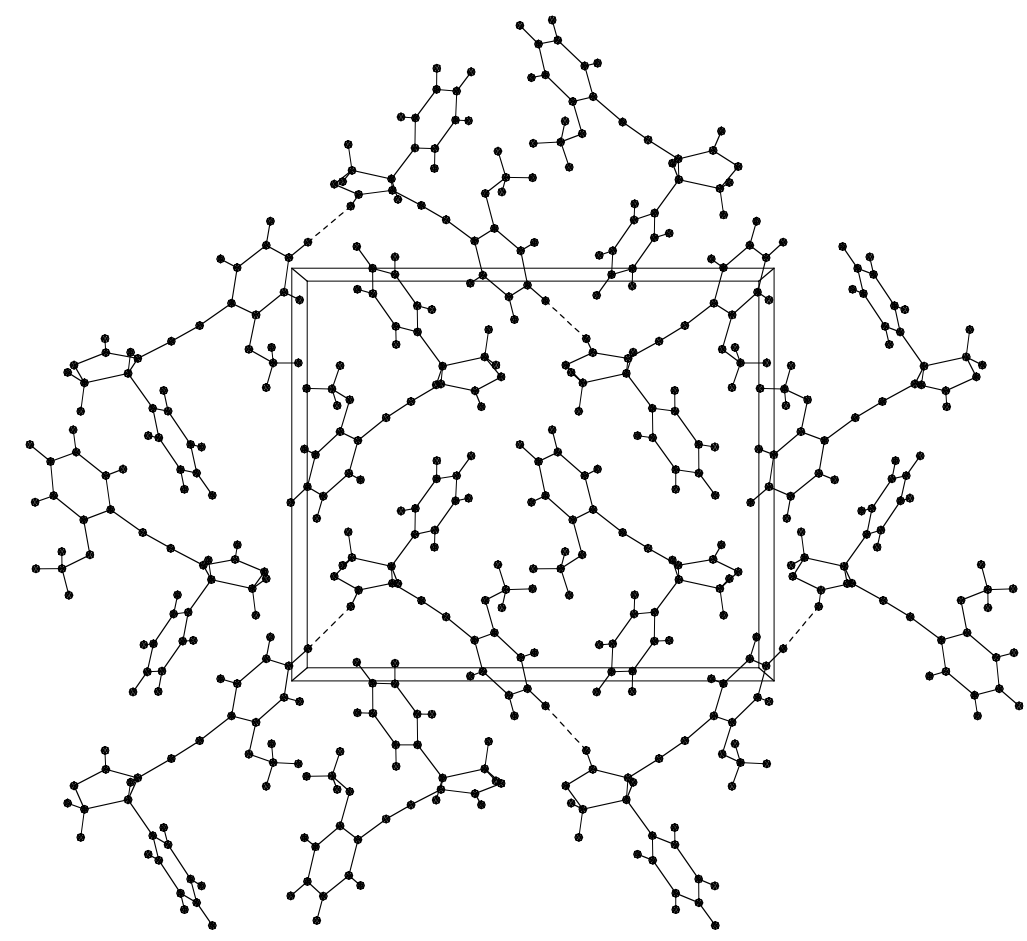




\section{Data collection}

A crystal (approximate dimensions $0.40 \times 0.12 \times 0.07 \mathrm{~mm}^{3}$ ) was placed onto the tip of a $0.1 \mathrm{~mm}$ diameter glass capillary and mounted on a CCD area detector diffractometer for a data collection at 173(2) K. A preliminary set of cell constants was calculated from reflections harvested from three sets of 20 frames. These initial sets of frames were oriented such that orthogonal wedges of reciprocal space were surveyed. This produced initial orientation matrices determined from 54 reflections. The data collection was carried out using MoKa radiation (graphite monochromator) with a frame time of 45 seconds and a detector distance of $4.9 \mathrm{~cm}$. A randomly oriented region of reciprocal space was surveyed to the extent of one sphere and to a resolution of $0.84 \AA$. Two major sections of frames were collected with $0.30^{\circ}$ steps in $\omega$ at four different $\phi$ settings and a detector position of $-28^{\circ}$ in $2 \theta$. The intensity data were corrected for absorption and decay (SADABS). ${ }^{1}$ Final cell constants were calculated from 1924 strong reflections from the actual data collection after integration (SAINT). ${ }^{2}$ Please refer to Table 1 for additional crystal and refinement information.

\section{Structure solution and refinement}

The structure was solved using Bruker SHELXTL ${ }^{3}$ and refined using Bruker SHELXTL. ${ }^{3}$ The space group P $2{ }_{1} 2_{1} 2_{1}$ was determined based on systematic absences and intensity statistics. A direct-methods solution was calculated which provided most non-hydrogen atoms from the E-map. Full-matrix least squares / difference Fourier cycles were performed which located the remaining non-hydrogen atoms. All non-hydrogen atoms were refined with anisotropic displacement parameters. All hydrogen atoms were placed in ideal positions and refined as riding atoms with relative isotropic displacement parameters. The final full matrix least squares refinement converged to $R 1=$ 0.0619 and $w R 2=0.1436\left(F^{2}\right.$, all data $)$.

\section{Structure description}

The structure is the one suggested. The specimen was a small needle, which contributed to a higher than expected R1.

Data collection and structure solution were conducted at the X-Ray Crystallographic Laboratory, 160 Kolthoff Hall, Department of Chemistry, University of Minnesota. All calculations were performed using Pentium computers using the current SHELXTL suite of programs. All publications arising from this report MUST either 1)include William W. Brennessel as a coauthor or 2)acknowledge William W. Brennessel, Victor G. Young, Jr., and the XRay Crystallographic Laboratory. 
1 An empirical correction for absorption anisotropy, R. Blessing, Acta Cryst. A51, 33-38 (1995).

2 SAINT V6.2, Bruker Analytical X-Ray Systems, Madison, WI (2001).

3 SHELXTL V6.10, Bruker Analytical X-Ray Systems, Madison, WI (2000).

4 A. Altomare, M. C. Burla, M. Camalli, G. Cascarano, C. Giacovazzo, A. Guagliardi, A. G. G. Moliterni, G.

Polidori, R. Spagna. Sir97: a new tool for crystal structure determination and refinement. J. Appl. Cryst. 32, 115119 (1998).

5 M. C. Burla, M. Camalli, B. Carrozzini, G. L. Cascarano, C. Giacovazzo, G. Polidori, R. Spagna. Sir2002: a new Direct Methods program for automatic solution and refinement of crystal structures. J. Appl. Cryst. (2003), in preparation.

6 A. L. Spek, Acta. Cryst. A46, C34 (1990). PLATON, A Multipurpose Crystallographic Tool, Utrecht University, Utrecht, The Netherlands, A. L. Spek (2000).

Some equations of interest:

$$
\begin{gathered}
R_{\text {int }}=\Sigma\left|F_{\mathrm{o}}^{2}-<F_{\mathrm{o}}^{2}>\right| / \Sigma\left|F_{\mathrm{o}}{ }^{2}\right| \\
R_{1}=\Sigma|| F_{\mathrm{o}}|-| F_{\mathrm{c}} \| / \Sigma\left|F_{\mathrm{o}}\right| \\
w R 2=\left[\Sigma\left[w\left(F_{\mathrm{o}}{ }^{2}-F_{\mathrm{c}}{ }^{2}\right)^{2}\right] / \Sigma\left[w\left(F_{\mathrm{o}}{ }^{2}\right)^{2}\right]\right]^{1 / 2} \\
\text { where } w=q /\left[\sigma^{2}\left(F_{\mathrm{o}}^{2}\right)+\left(a^{*} P\right)^{2}+b^{*} P+d+e^{*} \sin (\theta)\right] \\
\text { GooF }=S=\left[\Sigma\left[w\left(F_{\mathrm{o}}{ }^{2}-F_{\mathrm{c}}{ }^{2}\right)^{2}\right] /(n-p)\right]^{1 / 2}
\end{gathered}
$$


Table 1. Crystal data and structure refinement for $03254 \mathrm{a}$.

\begin{tabular}{|c|c|c|}
\hline Identification code & \multicolumn{2}{|l|}{$03254 a$} \\
\hline Empirical formula & \multicolumn{2}{|l|}{$\mathrm{C}_{18} \mathrm{H}_{15} \mathrm{~N} \mathrm{O}_{3}$} \\
\hline Formula weight & \multicolumn{2}{|l|}{293.31} \\
\hline Temperature & \multicolumn{2}{|l|}{$173(2) \mathrm{K}$} \\
\hline Wavelength & \multicolumn{2}{|l|}{$0.71073 \AA$} \\
\hline Crystal system & \multicolumn{2}{|l|}{ Orthorhombic } \\
\hline Space group & \multicolumn{2}{|l|}{$\mathrm{P} 2{ }_{1} 2_{1} 2_{1}$} \\
\hline \multirow[t]{3}{*}{ Unit cell dimensions } & $a=5.8632(13) \AA$ & $\alpha=90^{\circ}$ \\
\hline & $b=14.750(3) \AA$ & $\beta=90^{\circ}$ \\
\hline & $c=17.243(4) \AA$ & $\gamma=90^{\circ}$ \\
\hline Volume & \multicolumn{2}{|l|}{$1491.2(6) \AA^{3}$} \\
\hline$Z$ & \multicolumn{2}{|l|}{4} \\
\hline Density (calculated) & \multicolumn{2}{|l|}{$1.306 \mathrm{Mg} / \mathrm{m}^{3}$} \\
\hline Absorption coefficient & \multicolumn{2}{|l|}{$0.089 \mathrm{~mm}^{-1}$} \\
\hline$F(000)$ & \multicolumn{2}{|l|}{616} \\
\hline Crystal color, morphology & \multicolumn{2}{|l|}{ Colorless, Needle } \\
\hline Crystal size & \multicolumn{2}{|c|}{$0.40 \times 0.12 \times 0.07 \mathrm{~mm}^{3}$} \\
\hline Theta range for data collection & \multicolumn{2}{|l|}{1.82 to $25.06^{\circ}$} \\
\hline Index ranges & \multicolumn{2}{|c|}{$0 \leq h \leq 6,0 \leq k \leq 17,0 \leq l \leq 20$} \\
\hline Reflections collected & \multicolumn{2}{|l|}{7017} \\
\hline Independent reflections & \multicolumn{2}{|c|}{$1548[R($ int $)=0.0460]$} \\
\hline Observed reflections & \multicolumn{2}{|l|}{1405} \\
\hline Completeness to theta $=25.06^{\circ}$ & \multicolumn{2}{|l|}{$99.9 \%$} \\
\hline Absorption correction & \multicolumn{2}{|l|}{ Multi-scan } \\
\hline Max. and min. transmission & \multicolumn{2}{|l|}{0.9938 and 0.9651} \\
\hline Refinement method & \multicolumn{2}{|c|}{ Full-matrix least-squares on $F^{2}$} \\
\hline Data / restraints / parameters & \multicolumn{2}{|l|}{$1548 / 0 / 200$} \\
\hline Goodness-of-fit on $F^{2}$ & \multicolumn{2}{|l|}{1.120} \\
\hline Final $R$ indices $[I>2 \operatorname{sigma}(I)]$ & \multicolumn{2}{|c|}{$R 1=0.0619, w R 2=0.1393$} \\
\hline$R$ indices (all data) & \multicolumn{2}{|c|}{$R 1=0.0708, w R 2=0.1436$} \\
\hline Absolute structure parameter & \multicolumn{2}{|l|}{$-2(3)$} \\
\hline Largest diff. peak and hole & \multicolumn{2}{|c|}{0.196 and -0.218 e. $\AA^{-3}$} \\
\hline
\end{tabular}


Table 2. Atomic coordinates $\left(\mathrm{x} 10^{4}\right)$ and equivalent isotropic displacement parameters $\left(\AA^{2} \times 10^{3}\right)$ for 03254a. $U_{\text {eq }}$ is defined as one third of the trace of the orthogonalized $U_{i j}$ tensor.

\begin{tabular}{|c|c|c|c|c|}
\hline & $\mathrm{x}$ & $\mathrm{y}$ & $\mathrm{z}$ & $\mathrm{U}_{\mathrm{eq}}$ \\
\hline $\mathrm{O} 1$ & $4587(6)$ & $3297(2)$ & $3892(2)$ & $44(1)$ \\
\hline $\mathrm{O} 2$ & $7736(5)$ & $2599(2)$ & $4333(2)$ & $38(1)$ \\
\hline $\mathrm{O} 3$ & 2107(6) & $3093(2)$ & $1001(2)$ & $44(1)$ \\
\hline N1 & 7361(6) & $2767(2)$ & $3059(2)$ & $26(1)$ \\
\hline $\mathrm{C} 1$ & $6380(8)$ & 2924(3) & $3769(2)$ & $29(1)$ \\
\hline $\mathrm{C} 2$ & $9682(7)$ & 2371(3) & $3123(2)$ & $28(1)$ \\
\hline $\mathrm{C} 3$ & $9698(8)$ & 2147(3) & $3998(2)$ & $33(1)$ \\
\hline $\mathrm{C} 4$ & $10036(7)$ & $1549(2)$ & $2610(2)$ & $25(1)$ \\
\hline $\mathrm{C} 5$ & $12037(8)$ & $1460(3)$ & $2198(2)$ & $35(1)$ \\
\hline C6 & 12396(9) & $691(3)$ & $1738(3)$ & $46(1)$ \\
\hline $\mathrm{C} 7$ & 10756(9) & $32(3)$ & $1695(3)$ & $43(1)$ \\
\hline $\mathrm{C} 8$ & 8763(9) & $113(3)$ & 2118(3) & $48(1)$ \\
\hline C9 & $8392(8)$ & $869(3)$ & 2575(3) & $39(1)$ \\
\hline $\mathrm{C} 10$ & $6580(7)$ & $3192(3)$ & $2414(2)$ & $27(1)$ \\
\hline $\mathrm{C} 11$ & $5824(7)$ & $3586(3)$ & $1869(2)$ & $28(1)$ \\
\hline $\mathrm{C} 12$ & 4992(7) & $4150(3)$ & $1249(2)$ & $29(1)$ \\
\hline $\mathrm{C} 13$ & $3052(8)$ & $3916(3)$ & $815(2)$ & $32(1)$ \\
\hline $\mathrm{C} 14$ & 2225(9) & 4499(3) & $256(3)$ & $43(1)$ \\
\hline $\mathrm{C} 15$ & $3347(9)$ & $5310(3)$ & $114(3)$ & $44(1)$ \\
\hline $\mathrm{C} 16$ & $5305(9)$ & $5542(3)$ & $516(3)$ & $44(1)$ \\
\hline $\mathrm{C} 17$ & $6115(8)$ & 4971(3) & $1082(3)$ & $37(1)$ \\
\hline C18 & $201(8)$ & 2792(4) & $559(3)$ & $50(1)$ \\
\hline
\end{tabular}


Table 3. Bond lengths $[\AA]$ and angles $\left[^{\circ}\right]$ for 03254a.

\begin{tabular}{|c|c|c|c|}
\hline $\mathrm{O}(1)-\mathrm{C}(1)$ & $1.206(5)$ & $\mathrm{C}(7)-\mathrm{H}(7 \mathrm{~A})$ & 0.9500 \\
\hline $\mathrm{O}(2)-\mathrm{C}(1)$ & $1.345(5)$ & $C(8)-C(9)$ & $1.383(6)$ \\
\hline $\mathrm{O}(2)-\mathrm{C}(3)$ & $1.449(5)$ & $\mathrm{C}(8)-\mathrm{H}(8 \mathrm{~A})$ & 0.9500 \\
\hline $\mathrm{O}(3)-\mathrm{C}(13)$ & $1.372(5)$ & $\mathrm{C}(9)-\mathrm{H}(9 \mathrm{~A})$ & 0.9500 \\
\hline $\mathrm{O}(3)-\mathrm{C}(18)$ & $1.424(5)$ & $C(10)-C(11)$ & $1.189(5)$ \\
\hline $\mathrm{N}(1)-\mathrm{C}(10)$ & $1.356(5)$ & $C(11)-C(12)$ & $1.441(6)$ \\
\hline $\mathrm{N}(1)-\mathrm{C}(1)$ & $1.372(5)$ & $C(12)-C(13)$ & $1.405(6)$ \\
\hline $\mathrm{N}(1)-\mathrm{C}(2)$ & $1.485(5)$ & $C(12)-C(17)$ & $1.408(6)$ \\
\hline$C(2)-C(4)$ & $1.515(5)$ & C(13)-C(14) & $1.379(6)$ \\
\hline$C(2)-C(3)$ & $1.543(6)$ & $C(14)-C(15)$ & $1.386(7)$ \\
\hline $\mathrm{C}(2)-\mathrm{H}(2 \mathrm{~A})$ & 1.0000 & $\mathrm{C}(14)-\mathrm{H}(14 \mathrm{~A})$ & 0.9500 \\
\hline $\mathrm{C}(3)-\mathrm{H}(3 \mathrm{~A})$ & 0.9900 & $C(15)-C(16)$ & $1.384(7)$ \\
\hline $\mathrm{C}(3)-\mathrm{H}(3 \mathrm{~B})$ & 0.9900 & $\mathrm{C}(15)-\mathrm{H}(15 \mathrm{~A})$ & 0.9500 \\
\hline$C(4)-C(5)$ & $1.378(6)$ & $C(16)-C(17)$ & $1.374(6)$ \\
\hline$C(4)-C(9)$ & $1.391(6)$ & $\mathrm{C}(16)-\mathrm{H}(16 \mathrm{~A})$ & 0.9500 \\
\hline$C(5)-C(6)$ & $1.400(6)$ & $\mathrm{C}(17)-\mathrm{H}(17 \mathrm{~A})$ & 0.9500 \\
\hline $\mathrm{C}(5)-\mathrm{H}(5 \mathrm{~A})$ & 0.9500 & $\mathrm{C}(18)-\mathrm{H}(18 \mathrm{~A})$ & 0.9800 \\
\hline$C(6)-C(7)$ & $1.370(7)$ & $\mathrm{C}(18)-\mathrm{H}(18 \mathrm{~B})$ & 0.9800 \\
\hline $\mathrm{C}(6)-\mathrm{H}(6 \mathrm{~A})$ & 0.9500 & $\mathrm{C}(18)-\mathrm{H}(18 \mathrm{C})$ & 0.9800 \\
\hline$C(7)-C(8)$ & $1.382(7)$ & & \\
\hline $\mathrm{C}(1)-\mathrm{O}(2)-\mathrm{C}(3)$ & $110.2(3)$ & $\mathrm{C}(3)-\mathrm{C}(2)-\mathrm{H}(2 \mathrm{~A})$ & 110.0 \\
\hline $\mathrm{C}(13)-\mathrm{O}(3)-\mathrm{C}(18)$ & $117.9(4)$ & $\mathrm{O}(2)-\mathrm{C}(3)-\mathrm{C}(2)$ & $106.7(3)$ \\
\hline $\mathrm{C}(10)-\mathrm{N}(1)-\mathrm{C}(1)$ & $120.8(3)$ & $\mathrm{O}(2)-\mathrm{C}(3)-\mathrm{H}(3 \mathrm{~A})$ & 110.4 \\
\hline $\mathrm{C}(10)-\mathrm{N}(1)-\mathrm{C}(2)$ & $123.6(3)$ & $\mathrm{C}(2)-\mathrm{C}(3)-\mathrm{H}(3 \mathrm{~A})$ & 110.4 \\
\hline C(1)-N(1)-C(2) & $112.5(3)$ & $\mathrm{O}(2)-\mathrm{C}(3)-\mathrm{H}(3 \mathrm{~B})$ & 110.4 \\
\hline $\mathrm{O}(1)-\mathrm{C}(1)-\mathrm{O}(2)$ & $123.4(4)$ & $\mathrm{C}(2)-\mathrm{C}(3)-\mathrm{H}(3 \mathrm{~B})$ & 110.4 \\
\hline $\mathrm{O}(1)-\mathrm{C}(1)-\mathrm{N}(1)$ & $126.8(4)$ & $\mathrm{H}(3 \mathrm{~A})-\mathrm{C}(3)-\mathrm{H}(3 \mathrm{~B})$ & 108.6 \\
\hline $\mathrm{O}(2)-\mathrm{C}(1)-\mathrm{N}(1)$ & $109.7(3)$ & $\mathrm{C}(5)-\mathrm{C}(4)-\mathrm{C}(9)$ & $119.9(4)$ \\
\hline N(1)-C(2)-C(4) & $113.4(3)$ & $C(5)-C(4)-C(2)$ & $119.6(4)$ \\
\hline N(1)-C(2)-C(3) & $99.4(3)$ & $C(9)-C(4)-C(2)$ & $120.5(4)$ \\
\hline $\mathrm{C}(4)-\mathrm{C}(2)-\mathrm{C}(3)$ & $113.5(3)$ & $C(4)-C(5)-C(6)$ & $119.8(4)$ \\
\hline $\mathrm{N}(1)-\mathrm{C}(2)-\mathrm{H}(2 \mathrm{~A})$ & 110.0 & $\mathrm{C}(4)-\mathrm{C}(5)-\mathrm{H}(5 \mathrm{~A})$ & 120.1 \\
\hline $\mathrm{C}(4)-\mathrm{C}(2)-\mathrm{H}(2 \mathrm{~A})$ & 110.0 & $\mathrm{C}(6)-\mathrm{C}(5)-\mathrm{H}(5 \mathrm{~A})$ & 120.1 \\
\hline
\end{tabular}




$\begin{array}{llll}\mathrm{C}(7)-\mathrm{C}(6)-\mathrm{C}(5) & 120.1(4) & \mathrm{C}(14)-\mathrm{C}(13)-\mathrm{C}(12) & 120.2(4) \\ \mathrm{C}(7)-\mathrm{C}(6)-\mathrm{H}(6 \mathrm{~A}) & 120.0 & \mathrm{C}(13)-\mathrm{C}(14)-\mathrm{C}(15) & 119.6(4) \\ \mathrm{C}(5)-\mathrm{C}(6)-\mathrm{H}(6 \mathrm{~A}) & 120.0 & \mathrm{C}(13)-\mathrm{C}(14)-\mathrm{H}(14 \mathrm{~A}) & 120.2 \\ \mathrm{C}(6)-\mathrm{C}(7)-\mathrm{C}(8) & 120.2(4) & \mathrm{C}(15)-\mathrm{C}(14)-\mathrm{H}(14 \mathrm{~A}) & 120.2 \\ \mathrm{C}(6)-\mathrm{C}(7)-\mathrm{H}(7 \mathrm{~A}) & 119.9 & \mathrm{C}(16)-\mathrm{C}(15)-\mathrm{C}(14) & 121.2(4) \\ \mathrm{C}(8)-\mathrm{C}(7)-\mathrm{H}(7 \mathrm{~A}) & 119.9 & \mathrm{C}(16)-\mathrm{C}(15)-\mathrm{H}(15 \mathrm{~A}) & 119.4 \\ \mathrm{C}(7)-\mathrm{C}(8)-\mathrm{C}(9) & 120.2(5) & \mathrm{C}(14)-\mathrm{C}(15)-\mathrm{H}(15 \mathrm{~A}) & 119.4 \\ \mathrm{C}(7)-\mathrm{C}(8)-\mathrm{H}(8 \mathrm{~A}) & 119.9 & \mathrm{C}(17)-\mathrm{C}(16)-\mathrm{C}(15) & 119.4(5) \\ \mathrm{C}(9)-\mathrm{C}(8)-\mathrm{H}(8 \mathrm{~A}) & 119.9 & \mathrm{C}(17)-\mathrm{C}(16)-\mathrm{H}(16 \mathrm{~A}) & 120.3 \\ \mathrm{C}(8)-\mathrm{C}(9)-\mathrm{C}(4) & 119.8(4) & \mathrm{C}(15)-\mathrm{C}(16)-\mathrm{H}(16 \mathrm{~A}) & 120.3 \\ \mathrm{C}(8)-\mathrm{C}(9)-\mathrm{H}(9 \mathrm{~A}) & 120.1 & \mathrm{C}(16)-\mathrm{C}(17)-\mathrm{C}(12) & 120.7(5) \\ \mathrm{C}(4)-\mathrm{C}(9)-\mathrm{H}(9 \mathrm{~A}) & 120.1 & \mathrm{C}(16)-\mathrm{C}(17)-\mathrm{H}(17 \mathrm{~A}) & 119.6 \\ \mathrm{C}(11)-\mathrm{C}(10)-\mathrm{N}(1) & 176.9(4) & \mathrm{C}(12)-\mathrm{C}(17)-\mathrm{H}(17 \mathrm{~A}) & 119.6 \\ \mathrm{C}(10)-\mathrm{C}(11)-\mathrm{C}(12) & 173.9(4) & \mathrm{O}(3)-\mathrm{C}(18)-\mathrm{H}(18 \mathrm{~A}) & 109.5 \\ \mathrm{C}(13)-\mathrm{C}(12)-\mathrm{C}(17) & 118.8(4) & \mathrm{O}(3)-\mathrm{C}(18)-\mathrm{H}(18 \mathrm{~B}) & 109.5 \\ \mathrm{C}(13)-\mathrm{C}(12)-\mathrm{C}(11) & 121.9(4) & \mathrm{H}(18 \mathrm{~A})-\mathrm{C}(18)-\mathrm{H}(18 \mathrm{~B}) & 109.5 \\ \mathrm{C}(17)-\mathrm{C}(12)-\mathrm{C}(11) & 119.4(4) & \mathrm{O}(3)-\mathrm{C}(18)-\mathrm{H}(18 \mathrm{C}) & 109.5 \\ \mathrm{O}(3)-\mathrm{C}(13)-\mathrm{C}(14) & 125.0(4) & \mathrm{H}(18 \mathrm{~A})-\mathrm{C}(18)-\mathrm{H}(18 \mathrm{C}) & 109.5 \\ \mathrm{O}(3)-\mathrm{C}(13)-\mathrm{C}(12) & 114.8(4) & \mathrm{H}(18 \mathrm{~B})-\mathrm{C}(18)-\mathrm{H}(18 \mathrm{C}) & 109.5 \\ & & & \end{array}$

Symmetry transformations used to generate equivalent atoms: 
Table 4. Anisotropic displacement parameters $\left(\AA^{2} \times 10^{3}\right)$ for 03254a. The anisotropic displacement factor exponent takes the form: $-2 \pi^{2}\left[\mathrm{~h}^{2} \mathrm{a}^{* 2} \mathrm{U}_{11}+\ldots+2 \mathrm{~h} \mathrm{k} \mathrm{a*} \mathrm{b}^{*} \mathrm{U}_{12}\right]$

\begin{tabular}{|c|c|c|c|c|c|c|}
\hline & $\mathrm{U}_{11}$ & $\mathrm{U}_{22}$ & $\mathrm{U}_{33}$ & $\mathrm{U}_{23}$ & $\mathrm{U}_{13}$ & $\mathrm{U}_{12}$ \\
\hline $\mathrm{O} 1$ & $34(2)$ & $45(2)$ & $52(2)$ & $-2(2)$ & $10(2)$ & $8(2)$ \\
\hline $\mathrm{O} 2$ & $43(2)$ & $42(2)$ & $28(2)$ & $-2(1)$ & $3(1)$ & $4(2)$ \\
\hline $\mathrm{O} 3$ & $37(2)$ & $41(2)$ & $55(2)$ & $3(2)$ & $-14(2)$ & $-7(2)$ \\
\hline N1 & $27(2)$ & $26(2)$ & $24(2)$ & $2(1)$ & $0(2)$ & $4(2)$ \\
\hline $\mathrm{C} 1$ & $31(2)$ & $23(2)$ & $34(2)$ & $-2(2)$ & $1(2)$ & $-2(2)$ \\
\hline $\mathrm{C} 2$ & $23(2)$ & $27(2)$ & $33(2)$ & $4(2)$ & $-4(2)$ & $-1(2)$ \\
\hline C3 & $32(2)$ & $31(2)$ & $35(2)$ & $-4(2)$ & $-6(2)$ & $0(2)$ \\
\hline $\mathrm{C} 4$ & $24(2)$ & $25(2)$ & $25(2)$ & $5(2)$ & $-3(2)$ & $3(2)$ \\
\hline C5 & $30(2)$ & $36(2)$ & $40(2)$ & $3(2)$ & $5(2)$ & $1(2)$ \\
\hline C6 & $45(3)$ & $54(3)$ & $41(3)$ & $-1(2)$ & $9(2)$ & $16(3)$ \\
\hline C7 & $52(3)$ & $34(2)$ & $43(3)$ & $-7(2)$ & $-10(2)$ & $17(2)$ \\
\hline $\mathrm{C} 8$ & $47(3)$ & $30(2)$ & $65(4)$ & $-10(2)$ & $-5(3)$ & $-6(2)$ \\
\hline C9 & $31(2)$ & $27(2)$ & $57(3)$ & $-10(2)$ & $8(2)$ & $0(2)$ \\
\hline $\mathrm{C} 10$ & $25(2)$ & $23(2)$ & $32(2)$ & $-8(2)$ & $-2(2)$ & $-1(2)$ \\
\hline $\mathrm{C} 11$ & $25(2)$ & $29(2)$ & $30(2)$ & $-5(2)$ & $2(2)$ & $5(2)$ \\
\hline $\mathrm{C} 12$ & $30(2)$ & $30(2)$ & $28(2)$ & $0(2)$ & $3(2)$ & $10(2)$ \\
\hline $\mathrm{C} 13$ & $33(2)$ & $34(2)$ & $31(2)$ & $-4(2)$ & $-2(2)$ & $3(2)$ \\
\hline C14 & $39(3)$ & $57(3)$ & $33(2)$ & $-3(2)$ & $-5(2)$ & $16(3)$ \\
\hline $\mathrm{C} 15$ & $55(3)$ & $44(3)$ & $34(3)$ & $13(2)$ & $6(2)$ & $18(3)$ \\
\hline $\mathrm{C} 16$ & $51(3)$ & $33(2)$ & $48(3)$ & $8(2)$ & $5(3)$ & $7(2)$ \\
\hline C17 & $41(3)$ & $32(2)$ & $38(2)$ & $2(2)$ & $-2(2)$ & $4(2)$ \\
\hline $\mathrm{C} 18$ & $36(3)$ & $59(3)$ & $55(3)$ & $-11(3)$ & $-12(3)$ & $0(3)$ \\
\hline
\end{tabular}


Table 5. Hydrogen coordinates $\left(\mathrm{x} 10^{4}\right)$ and isotropic displacement parameters $\left(\AA^{2} \times 10^{3}\right)$ for 03254a.

\begin{tabular}{lrrrr}
\hline & $\mathrm{x}$ & $\mathrm{y}$ & $\mathrm{z}$ & $\mathrm{U}(\mathrm{eq})$ \\
\hline & & & & \\
H2A & 10858 & 2842 & 3006 & 33 \\
H3A & 9593 & 1484 & 4079 & 40 \\
H3B & 11122 & 2369 & 4241 & 40 \\
H5A & 13170 & 1920 & 2226 & 42 \\
H6A & 13777 & 627 & 1456 & 56 \\
H7A & 10989 & -483 & 1374 & 51 \\
H8A & 7644 & -352 & 2094 & 57 \\
H9A & 7019 & 925 & 2863 & 46 \\
H14A & 895 & 4346 & -30 & 52 \\
H15A & 2760 & 5713 & -266 & 53 \\
H16A & 6082 & 6091 & 401 & 53 \\
H17A & 7447 & 5132 & 1364 & 44 \\
H18A & -230 & 2180 & 725 & 75 \\
H18B & 607 & 2782 & 8 & 75 \\
H18C & -1084 & 3206 & 640 & 75 \\
& & & & \\
\hline & & & & \\
\hline
\end{tabular}


Table 6. Torsion angles $\left[{ }^{\circ}\right]$ for $03254 \mathrm{a}$.

\begin{tabular}{|c|c|c|c|}
\hline $\mathrm{C} 3-\mathrm{O} 2-\mathrm{C} 1-\mathrm{O} 1$ & $176.9(4)$ & C7-C8-C9-C4 & $-0.2(7)$ \\
\hline $\mathrm{C} 3-\mathrm{O} 2-\mathrm{C} 1-\mathrm{N} 1$ & $-3.4(4)$ & C5-C4-C9-C8 & $-0.8(7)$ \\
\hline C10-N1-C1-O1 & $13.5(6)$ & $\mathrm{C} 2-\mathrm{C} 4-\mathrm{C} 9-\mathrm{C} 8$ & $-178.5(4)$ \\
\hline C2-N1-C1-O1 & $174.4(4)$ & C1-N1-C10-C11 & $3(8)$ \\
\hline C10-N1-C1-O2 & $-166.1(3)$ & C2-N1-C10-C11 & $-156(8)$ \\
\hline C2-N1-C1-O2 & $-5.2(4)$ & N1-C10-C11-C12 & $62(11)$ \\
\hline C10-N1-C2-C4 & $-68.3(4)$ & C10-C11-C12-C13 & $-149(4)$ \\
\hline C1-N1-C2-C4 & $131.4(4)$ & $\mathrm{C} 10-\mathrm{C} 11-\mathrm{C} 12-\mathrm{C} 17$ & $30(4)$ \\
\hline C10-N1-C2-C3 & $170.9(3)$ & C18-O3-C13-C14 & $3.5(6)$ \\
\hline C1-N1-C2-C3 & $10.6(4)$ & C18-O3-C13-C12 & $-177.0(4)$ \\
\hline $\mathrm{C} 1-\mathrm{O} 2-\mathrm{C} 3-\mathrm{C} 2$ & $10.2(4)$ & $\mathrm{C} 17-\mathrm{C} 12-\mathrm{C} 13-\mathrm{O} 3$ & $178.1(4)$ \\
\hline $\mathrm{N} 1-\mathrm{C} 2-\mathrm{C} 3-\mathrm{O} 2$ & $-11.9(4)$ & $\mathrm{C} 11-\mathrm{C} 12-\mathrm{C} 13-\mathrm{O} 3$ & $-3.4(5)$ \\
\hline $\mathrm{C} 4-\mathrm{C} 2-\mathrm{C} 3-\mathrm{O} 2$ & $-132.6(3)$ & C17-C12-C13-C14 & $-2.4(6)$ \\
\hline N1-C2-C4-C5 & $136.9(4)$ & C11-C12-C13-C14 & $176.1(4)$ \\
\hline $\mathrm{C} 3-\mathrm{C} 2-\mathrm{C} 4-\mathrm{C} 5$ & $-110.7(4)$ & O3-C13-C14-C15 & $-179.2(4)$ \\
\hline N1-C2-C4-C9 & $-45.4(5)$ & C12-C13-C14-C15 & $1.3(6)$ \\
\hline C3-C2-C4-C9 & $67.0(5)$ & C13-C14-C15-C16 & $0.9(7)$ \\
\hline C9-C4-C5-C6 & $0.7(6)$ & C14-C15-C16-C17 & $-2.0(7)$ \\
\hline $\mathrm{C} 2-\mathrm{C} 4-\mathrm{C} 5-\mathrm{C} 6$ & $178.4(4)$ & C15-C16-C17-C12 & $0.9(7)$ \\
\hline C4-C5-C6-C7 & $0.4(7)$ & C13-C12-C17-C16 & $1.3(6)$ \\
\hline C5-C6-C7-C8 & $-1.4(7)$ & C11-C12-C17-C16 & $-177.2(4)$ \\
\hline C6-C7-C8-C9 & $1.3(7)$ & & \\
\hline
\end{tabular}

Symmetry transformations used to generate equivalent atoms: 